Original Article

\title{
The relationship between non-affected forelimb exercise and recovery after focal cerebral ischemia in acute phase
}

\author{
SAng-Su Na, PT, PhD ${ }^{1)}$, Seung-Kyu KIm, PT, MS ${ }^{1}$, NAm-Jin Jung, PT, MS ${ }^{1)}$, \\ GAK HwangBo, $\mathrm{PhD}^{1)^{*}}$ \\ 1) Department of Rehabilitation Science, Graduate School, Daegu University: 201 Daegudae-ro, \\ Jillyang, Gyeongsan-si, Gyeongsangbuk-do, Republic of Korea
}

\begin{abstract}
Purpose] In the present study, we hypothesized that exercise of the nonaffected forelimb in the early poststroke phase would stimulate the intact hemisphere, thereby influencing the hemisphere of the infarcted side and improving the performance of the hemiplegic limb. [Subjects and Methods] Adult male Sprague-Dawley rats (8-10 weeks of age, weighing 250-300 g, $\mathrm{n}=12$ ) were used and randomly divided into 3 groups: nonaffected forelimb exercise for 3 days and treadmill exercise 7 days after ischemia $(E T F, n=6)$, resting for 3 days and treadmill exercise 7 days after ischemia (ETN, $n=6$ ), and after ischemia 10 days resting group. To validate nerve growth factor (NGF), western blot analysis was performed. The results were analyzed using SPSS for Windows version 18.0. and expressed as mean \pm standard deviation (SD). [Results] Early treadmill exercise increased the expression of NGF protein level in both ETE and ETN groups. Comparing between the nonaffected forelimb exercise and infarct hemisphere in NGF protein expression, the ETE group showed higher increase of NGF protein level in right hemisphere than ETN group, but there was no statistical significance. [Conclusion] The early treadmill exercise increased NGF protein expression levels in both hemispheres and the nonaffected forelimb exercise in the early poststroke recovery phase could enhance neuronal recovery after focal ischemia in rat models.

Key words: Focal cerebral ischemia, Early phase exercise, Nerve growth factor
\end{abstract}

(This article was submitted Jun. 30, 2017, and was accepted Jul. 14, 2017)

\section{INTRODUCTION}

A stroke is a destructive cerebrovascular disease, which results in neurological deficits and long-term physical disabilities. Despite major advances in medical technology in recent years, most stroke survivors experience motor and cognitive dysfunction, as well as other neurological deficits ${ }^{1)}$.

Exercise interventions can enhance recovery after a stroke by direct electrical stimulation of the brain, which promotes the brain's intrinsic plasticity mechanisms (i.e., neuroplasticity) ${ }^{2}$. Several recent studies demonstrated beneficial effects of exercise and training in the early poststroke phase on ischemic brain recovery ${ }^{3,4}$. These effects are thought to be due to exercise increasing the activation of motor cortical areas in the undamaged hemisphere, which, in turn, compensates for nonactivation in areas that are damaged or disconnected.

The contralesional intact cerebral hemisphere contributes to motor function recovery in stroke patients ${ }^{5,6)}$. A previous study showed that the corticospinal tract (CST) was the main pathway for voluntary movement and that this pathway was connected to motor neurons in the spinal cord, either directly or indirectly ${ }^{7}$. In the study, the authors reported that the midline crossing CST fibers originated from the contralesional hemisphere correlated with behavioral outcomes after middle cerebral

*Corresponding author. Gak Hwangbo (E-mail: hbgak@daegu.ac.kr)

(C2017 The Society of Physical Therapy Science. Published by IPEC Inc.

(c) (1) $\odot$ This is an open-access article distributed under the terms of the Creative Commons Attribution Non-Commercial No Deriva-

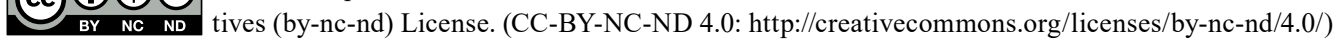


artery occlusion (MCAO) in an animal model ${ }^{7}$. Therefore, in the present study, we hypothesized that exercise of the nonaffected forelimb in the early poststroke phase would stimulate the intact hemisphere, thereby influencing the hemisphere of the infarcted side and improving the performance of the hemiplegic limb.

\section{SUBJECTS AND METHODS}

Experimental procedures were performed according to the protocols established by the Institution of Animal Care and Use Committee (IACUC) at the Daegu University, based on the NIH Guidelines for the Care and Use of Laboratory Animals (NIH, 1996).

Transient focal cerebral ischemia was induced by intraluminal occlusion of the right middle cerebral artery (MCA $)^{8}$. Briefly, middle cerebral artery is occluded by a $4-0$ nylon monofilament coated with a silicone tip, the monofilament was inserted into the internal carotid artery from the external carotid artery until mild resistance was felt. Reperfusion was established by completely withdrawing the nylon monofilament after $120 \mathrm{~min}$ of occlusion.

Adult male Sprague-Dawley rats (8-10 weeks of age, weighing 250-300 g, n=18) were used and randomly divided into 3 groups: non-affected forelimb exercise for 3 days and treadmill exercise 7 days after ischemia (ETF, $n=6$ ), resting for 3 days and treadmill exercise 7days after ischemia $(\mathrm{ETN}, \mathrm{n}=6)$, and after ischemia 10 days resting group (Control, $\mathrm{n}=6$ ).

Early non-affected side exercise in early phase was performed by skilled reach task. Skilled reach task as known as beneficial effect on neurotrophic factor expression that were conducted in a Plexiglas chamber containing of a $1 \mathrm{~cm} \mathrm{by} 10 \mathrm{~cm}$ window in front wall which is allowed for one of the rat's paw to reach through for a pellet ${ }^{9}$. . Sugar-flavored food pellets placed on the shelf placed in a small indentation $3 \mathrm{~cm}$ from the inside wall of the chamber and trained in 30 minutes per 1 day for 3 days based on previous reports ${ }^{10,11}$. The pellets are aligned the right window side for nonaffected forelimb exercise.

The validate nerve growth factor (NGF), western blot analysis was performed. In brief, proteins extracts from brain tissues that were divided right/left hemisphere and separated by sodium dodecyl sulfate-polyacryamide gel electrophoresis. Protein separation was performed then transferred to nitrocellulose, and the blots were probed with anti-NGF 1:1,000 (E-12, cat\# sc-365944, Santa Cruz, CA, USA). Peroxidase anti-mouse was used as a secondary antibody. The thickness of the bands was photographically measured by Scion Image software Beta 4.0.3 (Scion Corp., Frederick, MD, USA). The results were analyzed using SPSS for Windows version 18.0. and expressed as mean \pm standard deviation (SD). Comparisons between groups were performed via the Bonferroni-Dunn test. $\mathrm{P}$ values less than 0.05 at the $95 \%$ confidence level were considered significant.

\section{RESULTS}

Early treadmill exercise increased the expression of NGF protein level in both ETE and ETN groups (Table 1). Two groups were a significantly increased NGF expression in both hemisphere than control group, but there was no significantly expression of NGF in ETE and ETN groups. Comparing between the non-affected forelimb exercise and infarct hemisphere in NGF protein expression, the ETE group showed higher increase of NGF protein level in right hemisphere than ETN group, but there was no statistical significance.

\section{DISCUSSION}

This study demonstrated that ETE of the nonaffected forelimb was correlated with ischemic recovery in rats in an MCAO

Table 1. NGF protein expression in focal ischemia rats brain ( $n=6$ per group)

\begin{tabular}{llc}
\hline & \multicolumn{2}{c}{ Relative optical density } \\
\hline \multirow{2}{*}{ Control } & Right side & $3,558.1 \pm 330.3$ \\
& Left side & $1,266 \pm 214.7$ \\
ETN & Right side & $7,269.5 \pm 291.8^{*}$ \\
& Left side & $4,986.8 \pm 247.9^{*}$ \\
ETF & Right side & $8,316.8 \pm 391.7^{*}$ \\
& Left side & $4,306.3 \pm 161.9^{*}$ \\
\hline
\end{tabular}

Data were presented as mean \pm SD.

*Significantly different from Control group

ETF: early treadmill exercise with nonaffected forelimb exercise group; ETN: early treadmill exercise with no forelimb exercise group; Right side: Infarct hemisphere; Left side: Intact hemisphere 
model. Furthermore, the findings suggested that ETE significantly increased the protein expression level of nerve growth factor (NGF) of both hemispheres of MCAO rats. Previous experimental rat models and clinical studies also demonstrated that rehabilitative exercise promoted neurological recovery and functional recovery after a stroke $e^{4,12)}$.

As mentioned earlier, high-intensity exercise or excessive use of the affected limb can exacerbate ischemic injury ${ }^{13)}$. However, moderate-intensity exercise, combined with an appropriate training protocol, can be an effective poststroke treatment. Therefore, in the present study, we investigated whether ETE exercise would improve ischemic recovery. As shown by the comparison between the ETE groups and the control group, the NGF protein expression level was significantly increased in the ETN and ETE groups as compared to that of the control group.

The corpus callosum (CC) is a white-matter structure and is the major commissure connecting the cerebral hemispheres. Thus, we hypothesized that the addition of nonaffected forelimb exercise in the early phase would influence both the infarcted hemisphere and intact hemisphere. The results showed that NGF protein expression was slightly increased in the ETN group as compared to that in the ETE group, but the finding was not statistically significant. The results do not explain the neurological mechanism underlying the correlation between nonaffected forelimb exercise and recovery of an infarcted hemisphere. However, in common with the findings of a previous study ${ }^{7}$, they point to the possibility of exercise having an influence on hemispheric connections ${ }^{7)}$.

In conclusion, the results of the present showed that early treadmill exercise increased NGF protein expression levels in both hemispheres and suggested that exercise of the nonaffected forelimb in the early poststroke recovery phase could enhance neuronal recovery after focal ischemia in rat models. Further studies are needed to provide neurological evidence of hemispheric communication and develop methods for a clinical approach in stroke patients.

\section{REFERENCES}

1) Saxena SK, $\mathrm{Ng} \mathrm{TP}$, Koh G, et al.: Is improvement in impaired cognition and depressive symptoms in post-stroke patients associated with recovery in activities of daily living? Acta Neurol Scand, 2007, 115: 339-346. [Medline] [CrossRef]

2) Johnston MV: Plasticity in the developing brain: implications for rehabilitation. Dev Disabil Res Rev, 2009, 15: 94-101. [Medline] [CrossRef]

3) Johansson BB: Brain plasticity and stroke rehabilitation. The Willis lecture. Stroke, 2000, 31: 223-230. [Medline] [CrossRef]

4) Yang YR, Wang RY, Wang PS: Early and late treadmill training after focal brain ischemia in rats. Neurosci Lett, 2003, 339: 91-94. [Medline] [CrossRef]

5) Johansen-Berg H, Rushworth MF, Bogdanovic MD, et al.: The role of ipsilateral premotor cortex in hand movement after stroke. Proc Natl Acad Sci USA, 2002, 99: 14518-14523. [Medline] [CrossRef]

6) Gerloff C, Bushara K, Sailer A, et al.: Multimodal imaging of brain reorganization in motor areas of the contralesional hemisphere of well recovered patients after capsular stroke. Brain, 2006, 129: 791-808. [Medline] [CrossRef]

7) Liu Z, Li Y, Zhang X, et al.: Contralesional axonal remodeling of the corticospinal system in adult rats after stroke and bone marrow stromal cell treatment. Stroke, 2008, 39: 2571-2577. [Medline] [CrossRef]

8) Zhang P, Zhang Q, Pu H, et al.: Very early-initiated physical rehabilitation protects against ischemic brain injury. Front Biosci (Elite Ed), 2012, 4: 2476-2489. [Medline]

9) Yong MS, Kim SG, Cheon SH: Effects of skilled reach training with affected forelimb and treadmill exercise on the expression of neurotrophic factor following ischemia-induced brain injury in rats. J Phys Ther Sci, 2017, 29: 647-650. [Medline] [CrossRef]

10) Girgis J, Merrett D, Kirkland S, et al.: Reaching training in rats with spinal cord injury promotes plasticity and task specific recovery. Brain, 2007, 130 : 2993-3003. [Medline] [CrossRef]

11) Maldonado MA, Allred RP, Felthauser EL, et al.: Motor skill training, but not voluntary exercise, improves skilled reaching after unilateral ischemic lesions of the sensorimotor cortex in rats. Neurorehabil Neural Repair, 2008, 22: 250-261. [Medline] [CrossRef]

12) Enzinger C, Dawes H, Johansen-Berg H, et al.: Brain activity changes associated with treadmill training after stroke. Stroke, 2009, 40: 2460-2467. [Medline] [CrossRef]

13) Bland ST, Schallert T, Strong R, et al.: Early exclusive use of the affected forelimb after moderate transient focal ischemia in rats: functional and anatomic outcome. Stroke, 2000, 31: 1144-1152. [Medline] [CrossRef] 